\title{
¿Cómo evalúan los docentes universitarios las asignaturas que facilitan?
}

\section{Información de artículo:}

Recibido: 03.04.2019

Aprobado: 22.05.2019

\section{Palabras claves:}

aprendizaje,

evaluación,

formas de evaluación,

intervención educativa.
Martha Miurel Suárez Soza ${ }^{1}$, Carmen María Triminio Zavala ${ }^{2}$, Ricardo Antonio Madriz Aguilar ${ }^{3}$, Eddy Roberto Silva Largaespada ${ }^{4}$, Eugenio Casimiro López Mairena ${ }^{5}$

\section{Resumen}

El objetivo de este trabajo era conocer las estrategias de evaluación utilizadas por los docentes de dos Facultades de la UNAN-Managua (Facultad de Educación e Idiomas RURD y Facultad Regional Multidisciplinaria FAREM-Estelí), en el proceso de aprendizaje; identificando los referentes teóricos-prácticos que guían el quehacer pedagógico y direccionan las formas de evaluación de acuerdo con la función, temporalidad e involucrados; para ello se desarrolló un diagnóstico de las experiencias desde la perspectiva de los docentes y estudiantes quienes coincidieron que el examen escrito es una de las estrategias de evaluación más utilizada sustentada en concepciones tradicionales que no conciben otras alternativas de evaluación. Posteriormente se realizó la intervención educativa centrada en los estudiantes, como una praxis que integra acción, práctica y reflexión crítica de una forma ordenada, mediante el intercambio colectivo de saberes, pensares, experiencias, actitudes y prácticas en los procesos de evaluación de los aprendizajes.

\footnotetext{
${ }^{1}$ Máster en Gestión del Desarrollo Comunitario. Coordinadora de Extensión Universiaria de la Facultad Multidisciplinaria de Estelí de la Universidad Nacional Autónoma de Nicaragua, Managua. Email: msuarezs@unan.edu.ni ำ: https://orcid. org/oooo-0oo2-1851-3774

2 Máster en Didáctica Específicas de las Matemáticas. Coordinadora de la Carrera de Física-Matemática de la Facultad Multidisciplinaria de Estelí de la Universidad Nacional Autónoma de Nicaragua, Managua. Email ctriminio@unan.edu.ni (D): https://orcid.org/oooo-0oo1-5970-5396

3 Licenciado en Administración Agropecuaria. Coordinador de la Disciplina de Atletismo de la Universidad Nacional Autónoma de Nicaragua, Managua-Recinto Universitario Rubén Darío. Email: ricardo.madriz@unan.edu.ni (1): https:// orcid.org/oooo-0003-3044-7762

4 Licenciado en Educación Física y Deportes. Coordinador de la Disciplina Taekwondo de la Universidad Nacional Autónoma de Nicaragua, Managua-Recinto Universitario Rubén Dario. Email: eddy.silva@unan.edu.ni (1): https://orcid. org/oooo-0002-1180-9804

5 Doctor en Innovación en la Formación. Vicerrector de la Universidad de las Regiones Autónomas de la Costa Caribe NicaragüenseRecinto Universitario Nueva Guinea. Email: eugenio.lopez@uraccan.edu.ni 우: https://orcid.org/oooo-ooo2-7929-7817
} 


\title{
How do university teachers assess the subjects they facilitate?
}

\begin{abstract}
The objective of this work was to know the assessment strategies used by the teachers of two Faculties of the UNAN-Managua (Faculty of Education and Languages RURD and Multidisciplinary Regional Faculty FAREM-Estelí), in the learning process; identifying the theoretical-practical references that guide the pedagogical work and direct the ways of assessment according to the function, temporality and involved; For this, a diagnosis of the experiences was developed from the perspective of teachers and students who agreed that the written exam is one of the most used evaluation strategies based on traditional conceptions that do not conceive of other evaluation alternatives. Subsequently, the student-centered educational intervention was carried out, as a praxis that integrates action, practice and critical reflection in an orderly manner, through the collective exchange of knowledge, thoughts, experiences, attitudes and practices in the assessment learning processes.
\end{abstract}

Keywords: learning, assessment, assessment ways, educational intervention.

\section{Introducción}

Las nuevas propuestas sobre la evaluación, producto de la transformación curricular, aparecen en el Modelo Educativo y en los Programas de Asignatura que están vigentes, implican la necesidad de hacer cambios en la forma tradicional.

Tomando en cuenta que la forma en qué se evalúa a los estudiantes afecta (positiva o negativamente) su proceso de aprendizaje; las estrategias de evaluación que los docentes aplican es un factor determinante, para garantizar la calidad del proceso educativo.

El solo hecho de nombrar la palabra evaluación en el salón de clases o referirse a que el contenido impartido será evaluado en la próxima clase, produce pánico en los estudiantes, considerado importante conocer las estrategias de evaluación utilizadas en el proceso de aprendizaje de acuerdo con los nuevos enfoques en el ámbito educativo.

Este estudio, favoreció el acercamiento con docentes y estudiantes para reflexionar sobre los cambios propuestos en el modelo educativo y su aplicación, valorar si permanecen en lo tradicional o sí realmente están haciendo cambios para mejorar y actualizar las formas de evaluación, mediante un ejercicio de intervención educativa. 


\section{INVESTIGACIÓN EDUCATIVA}

\section{Literatura}

Para Pimenta Prieto (2008) la evaluación es la emisión de juicio sistemático valorando las competencias adquiridas en un determinado contexto específico, el autor enfatiza en la importancia que tiene el ejercicio de observación realizada por los docentes en los procesos de interacción, que son la base cualitativa para los juicios valorativos (p.26).

Arias Lara y de Arias Peñaloza (2011) mencionan que:

Exponer un concepto único de la evaluación de los aprendizajes resulta una labor titánica e imposible de lograr; la diversidad de enfoques ha promovido en la literatura especializada el establecimiento de numerosas definiciones, esto aunado a la constante evolución del sentido del término dentro de los sistemas educativos (p.3).

Cano (2012) expresa que la evaluación está estrechamente vinculado con el aprendizaje, ya que la evaluación convierte las experiencias educativas en educación, instrucción y todo contribuyen con el desarrollo de competencias para desarrollo personal (p.11).

Según Santos Guerra (1999) "la evaluación pone de manifiesto todas nuestras concepciones docentes: lo que es la Universidad, la naturaleza del proceso de enseñanza aprendizaje, el papel del docente, la relación con el estudiante; se podría decir sin mucho temor a equivocarse: dime cómo evalúas y te diré qué tipo de profesional eres" (p. 2).

En el ámbito educativo el término objetivo, es el preámbulo del proceso de evaluación, Foronda Torrico y otros (2007) argumentan que son el marco de referencia en la dirección del proceso de evaluación como principios orientadores, que corrigen el camino del proceso educativo real, en función de lo planificado hasta llegar a discriminar resultados obtenidos (p.19).

Pimienta (2008) plantea que uno de los criterios de evaluación es el que ésta realiza con base a los objetivos y alcances que persigue el proceso educativo, destaca dos dimensiones: función sumativa y formativa; señala que la función sumativa se centra en el valor final de un proceso, con ello busca tomar decisiones múltiples como es promover o no a los estudiantes a un grado inmediato superior, cambiar libros de textos, ente otros (p. 33).

Najarro (2009) argumenta que el carácter pedagógico, de la evaluación formativa describe la función que permite informar al estudiantado, madres y padre de familia sobre los cambios, obstáculos y como deben ser superados para mejorar el proceso de aprendizaje. Desde esta perspectiva, la identificación de necesidades, ajustes y mejoras 
que surgen de las actividades que desarrolla el estudiante en su proceso de formación, es lo que permite darle seguridad y elevar su autoestima (p. 59).

El Modelo educativo (UNAN-Managua, 2011) plantea que el objetivo primordial no es la evaluación sumativa de los conocimientos adquiridos por los estudiantes; al contrario debe ser la reunión de información ordenada y sistemática que permite la toma de decisiones; creando espacios, ambientes e instrumentos que favorezcan la heteroevaluación, autoevaluación y coevaluación (pp.32-33).

Tobón, Pimienta y García (2010) definen que la evaluación de acuerdo a sus participantes e integra procesos personales y colectivos donde interactuan estudiantes y docentes, para juntos evaluar el proceso de aprendizaje y buscar alternativas de mejoras; aplicando la autoevaluación (personal), coevaluación (colectiva) y heteroevaluación (docente) (pp. 130-131).

Una evaluación integrada en el proceso de aprendizaje, evalúa todos los objetivos en un contexto realista, recogiendo evidencias de aprendizaje producidas por el estudiantado; asegura la coherencia entre objetivos, metodología docente y evaluación, y no solo de los resultados (Fernández Marcha, 2009, p.29).

Amaranti Pesce (2010) define la retroalimentación como acción crucial para transformar la evaluación en una oportunidad para aprender, dando relevancia a la comunicación de los resultados y las acciones que se proponen al estudiante para mejorar (p. 4).

En el modelo educativo de la UNAN-Managua (2011) se argumenta que las TIC'S se utilizarán no solo para mejorar las prácticas pedagógicas en el aula, sino también para permitirles al estudiantado otros escenarios de aprendizaje. Estas se integran como objeto de aprendizaje (planes de estudios), medio de aprendizaje o (facilitación del proceso) y herramienta del trabajo independiente (pp.33-34).

\section{Metodología de la investigación}

La intervención educativa implicó el desarrollo de cinco momentos metodológicos:

\begin{tabular}{|l|l|l|}
\hline Primer momento & $\begin{array}{l}\text { Planificación conceptual del proceso a } \\
\text { desarrollar }\end{array}$ & $\begin{array}{l}\text { Definición de objetivos y escenario de inter- } \\
\text { vención - acompañamiento } \\
\text { Definición de categorías de análisis y referen- } \\
\text { tes conceptuales }\end{array}$ \\
\hline Segundo momento & Construcción de metodología & $\begin{array}{l}\text { Definición, validación y digitalización de } \\
\text { instrumentos } \\
\text { Definición de participantes }\end{array}$ \\
\hline Tercer momento & Aplicación de instrumentos de diagnóstico \\
\hline Cuarto momento & Análisis de resultados de instrumentos de diagnostico \\
\hline
\end{tabular}




\section{INVESTIGACIÓN EDUCATIVA}

\begin{tabular}{|l|l|l|}
\hline Quinta momento & $\begin{array}{l}\text { Intervención educativa } \\
\text { Virtual }\end{array}$ & $\begin{array}{l}\text { Se construyeron dos grupos de intervención } \\
\text { educativa virtual en Facebook y Google } \\
\text { Classroom }\end{array}$ \\
\hline
\end{tabular}

Ilustración 1: Fuente propia. Momentos metodológicos

Este proceso se organizó a partir un sistema categorial que facilito la operativización y el análisis de los resultados.

Referentes para

planificar

estrategias de

evaluación
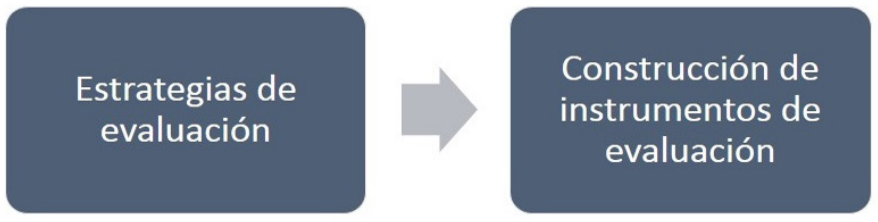

Ilustración 2: Fuente propia. Categorías de análisis

Para la definición de los instrumentos se consideró la realidad del escenario de intervención, los tiempos de aplicación y el aprovechamiento de las TICs (tecnologías de información y comunicación) accesibles para llegar a un grupo considerable de población docente y estudiantil que proporcionaran información de manera espontánea.

Cuestionario: contemplaba 9 ítems, con preguntas cerradas; se trabajó desde un software (e-encuesta) de encuestas en línea y herramientas de cuestionarios; se organizaron en 2 guías dirigidas a docentes y estudiantes.

Entrevista a profundidad: estructurada por 10 ítems, se abordaban aspectos diagnósticos y de mejoras en los procesos de evaluación según la experiencia práctica; se organizó 1 guía de entrevista a profundidad dirigida a docentes.

La muestra estuvo conformada por docentes y estudiantes:

\begin{tabular}{|l|l|l|l|}
\hline \multicolumn{1}{|c|}{ Facultades } & \multicolumn{1}{c|}{ Docentes } & \multicolumn{1}{c|}{ Estudiantes } & \multicolumn{1}{c|}{ Total } \\
\hline Educación e Idiomas - RURD & 13 & 35 & 48 \\
\hline FAREM-Estelí & 16 & 40 & 56 \\
\hline Total & 29 & 75 & 104 \\
\hline
\end{tabular}

Ilustración 3: Fuente propia. Participantes

\section{Resultados}

Los docentes mencionaron referentes teóricos y prácticos que guían su quehacer pedagógico, listando una seria de documentos que son fundamentales para el proceso de planificación didáctica y que inciden en la selección, integración y adecuación de 
estrategias de aprendizajes y de evaluación; refieren tres documentos que describen las estrategias de evaluación:

Plan didáctico: es el nivel más concreto de la planificación educativa, describe los momentos conceptuales, procedimentales y actitudinales; plasma las estrategias de aprendizaje y evaluación.

Planes de clase: instrumento teórico, metodológico y procedimental, no existen formatos y estructuras únicas.

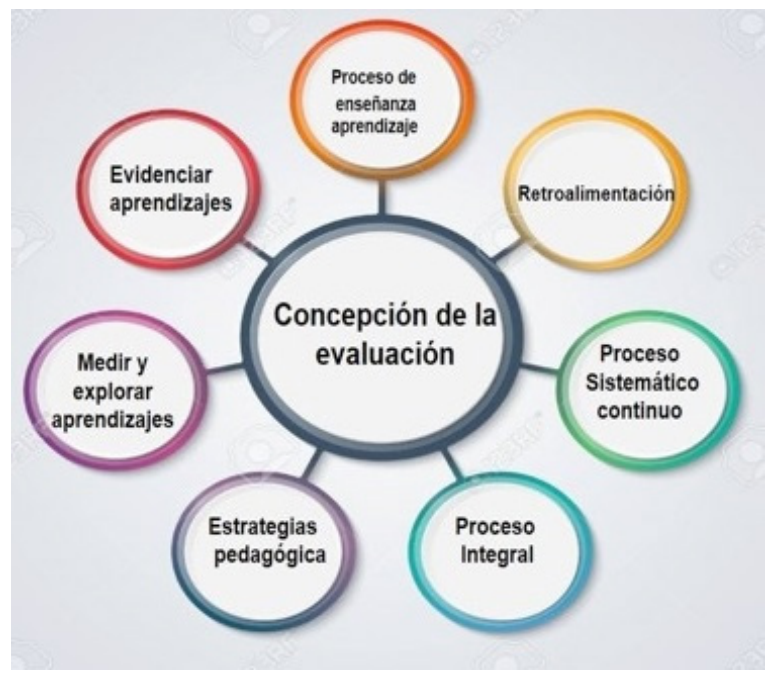

Ilustración 4 Fuente propia: Análisis Cognoscitivo

Dossier de la asignatura: es la recopilación, cronológicamente ordenada, de los materiales teóricos, metodológicos y procedimentales relevantes para el aprendizaje de los estudiantes.

Los docentes entrevistados definen la evaluación en 7 términos cognoscitivos que ilustran la experiencia desde su rol como docentes universitarios, vinculados a los propósitos y objetivos que persiguen en la planificación de los procesos de evaluación en la práctica pedagogía.

Es importante destacar que en sus análisis cognoscitivos refieren aspectos que determinan su comprensión, planificación y experiencia pedagógica, entre ellos: 


\begin{tabular}{|l|l|}
\hline $\begin{array}{l}\text { La evaluación condicionada por la formación disciplinar } \\
\text { de cada docente, las temáticas de trabajo; así como las } \\
\text { disciplinas científicas donde desempeñan su quehacer } \\
\text { pedagógico. }\end{array}$ & $\begin{array}{l}\text { "Es un proceso de enseñanza-aprendizaje, dependiendo del tema } \\
\text { que se estará impartiendo, se analiza el tema para ver que es- } \\
\text { trategias se deben usar para el proceso de evaluación." (Docente } \\
\text { \#5- entrevistada) }\end{array}$ \\
\hline $\begin{array}{l}\text { Los objetivos que se persiguen: mejorar, medir avances } \\
\text { y formación integral. }\end{array}$ & $\begin{array}{l}\text { "La evaluación es la retroalimentación del proceso de enseñanza- } \\
\text { aprendizaje; los datos recopilados sirven para mejorar las difi- } \\
\text { cultades que se presenten en la realización del proceso; permite } \\
\text { conocer los logros alcanzados y el nivel de aprendizaje que los } \\
\text { estudiantes han alcanzado en ese momento." (Docente \# 3- en- } \\
\text { trevistada) }\end{array}$ \\
\hline Seguimiento de los procesos de aprendizajes de los \\
estudiantes. & $\begin{array}{l}\text { “.. permite medir en cierta manera los planes ejecutados y } \\
\text { explorar los aprendizajes logrados por parte de los estudiantes, } \\
\text { así mismo permite al docente mejorar pequeños detalles que } \\
\text { pudieran afectar el proceso, ya que la evaluación al ser un proce- } \\
\text { so continuo hace que el educando mejore y también el docente." } \\
\text { (Docente \# 9- entrevistada) }\end{array}$ \\
\hline Diagnóstico, identificación de fortalezas y debilidades & $\begin{array}{l}\text { "... conjunto de estrategias pedagógicas que nos permite } \\
\text { identificar las fortalezas y debilidades y analizar los logros en el } \\
\text { desarrollo del proceso de enseñanza-aprendizaje." (Docente \# } \\
4 \text { - entrevistada) }\end{array}$ \\
\hline
\end{tabular}

Ilustración 5: Fuente propia. Citas textuales de docentes.

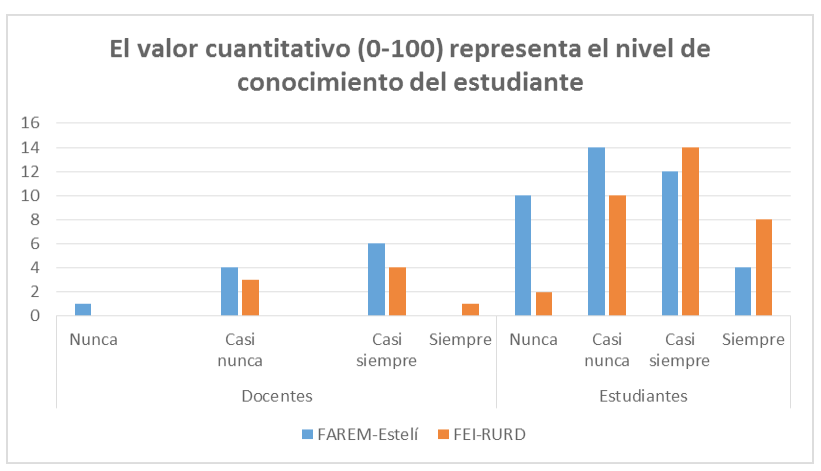

Ilustración 6: Fuente propia: Resultados de encuesta.

La encuesta realizada a docentes y a estudiantes, evidencia que ambos coincide que casi siempre la valoración cuantitativa (o-10o), refleja el nivel de conocimiento adquirido en el proceso de aprendizaje.

La concepción de la evaluación lleva a concretarse en una práctica cargada de subjetividad, donde la responsabilidad es asumida por el docente, quien se enfoca en los resultados cuantitativos y los cualitativos son la lectura de la cualitativa; perdiendo el valor complementario de las dos alternativas de evaluación como lo refiere (Najarro Arriola, 2009, p. 59). 


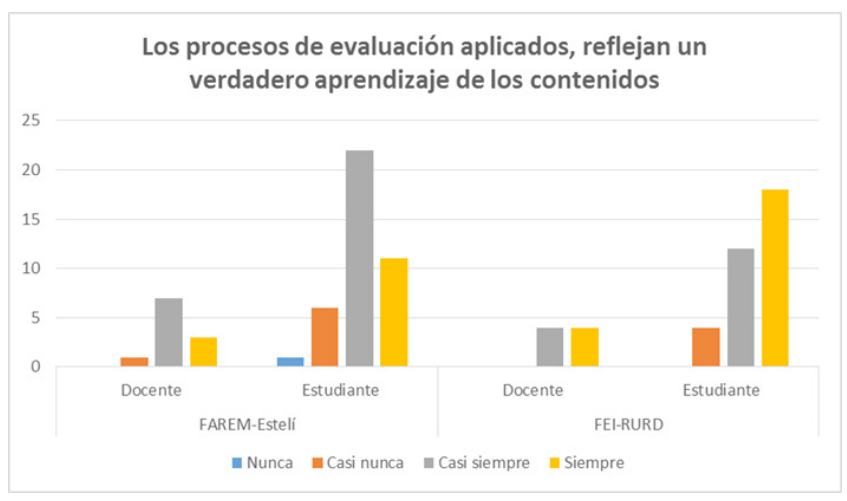

Ilustración 7: Fuente propia: Resultados de encuesta.

Vale la pena destacar que estudiantes y docentes reflejan que los procesos de aprendizaje, deben de estar estrechamente vinculadas a las estrategias y formas de evaluación que determinan que el proceso de aprendizaje sea pertinente, oportuno y respondan a las demandas del contexto.

La utilidad de los tipos y formas de evaluación está determinada por aspectos socioeducativos y curriculares de los procesos de aprendizaje, como son las características particulares de la asignatura, del grupo y perfil de la carrera.

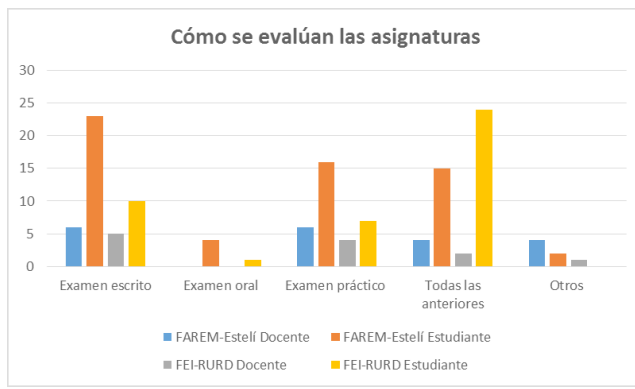

Ilustración 8: Fuente propia: Resultado de encuesta.

Las experiencias que se desarrollan en las aulas de clase, ponen de manifiesto que el examen escrito sigue siendo una de las estrategias de evaluación más utilizada en los procesos de aprendizaje, sobre todo cuando la práctica docente sigue sustentada en concepciones tradicionales de educación que no conciben otras alternativas de evaluación; situación que coincide con Rodríguez Gómez et al. (2013), que expone que los tradicionales exámenes son otra dificultad permanente de la educación superior, a pesar de los cambios en la evaluación de los aprendizajes (p. 199). 
Los docentes concuerdan que las estrategias de evaluación no son una acción espontanea, al contrario, responden a los resultados de los procesos de planificación educativa y pedagógica que caracterizan la formación académica, donde juega un papel fundamental la organización metodológica de los procesos de aprendizaje, estructurados por fundamentos institucionales, curriculares y disciplinares.

"Desde el momento que ya tengo las unidades y los temas a impartir de la asignatura, trabajo en los objetivos, los contenidos y sub-contenidos, después de esto ya puedo definir las formas y estrategias de evaluación a aplicar durante el semestre." (Docente \#9- Entrevistada)

Además refieren que la evaluación de aprendizajes tiene propósitos distintos para estudiantes y docentes; los estudiantes visualizan la asimilación, comprensión de aprendizajes y el desarrollo de capacidades; en el caso de los docentes se destacan las vinculadas al desempeño en el proceso de aprendizaje.

La evaluación como proceso tiene propósitos cualitativo-cuantitativo-cualitativo; cualitativos porque valora los conocimientos previos, la construcción del nuevo aprendizaje, así como la identificación de dificultades y alternativas de soluciones oportunas; cuantitativo permite cumplir con un resultado numérico como lo establecen las normativas de evaluación y se cumple con las disposiciones académico-administrativas; y cualitativo nuevamente porque los resultados deben de plantear una valoración, del ¿por qué? se obtuvo esa calificación numérica.

"Ambos (cualitativo y cuantitativo), ya que luego de valorar el aprendizaje asimilado, de analizar el desempeño y el desarrollo de capacidades, habilidades y competencias, debo comunicarles sobre sus necesidades de aprendizaje; para que realicen su mejor esfuerzo a fin de mejorar su aprendizaje, según sea el caso." (Docente \#10- Entrevistada)

La retroalimentación se desarrolla posterior a una evaluación aplicada, permite al estudiante conocer avances, aciertos, dificultades y buscar alternativas de mejora que motive y regule su autoaprendizaje:

\begin{tabular}{|l|l|}
\hline \multicolumn{1}{|c|}{ Atención personalizada } & \multicolumn{1}{|c|}{ Reunión con el grupo de clase, reforzamiento de contenidos. } \\
\hline "Depende de la asignatura, por ejemplo, en Informática & "Realizando plenario en el aula, donde se revisan pregunta por \\
Básica que se desarrolla en línea, la retroalimentación & pregunta, o según sea el caso. Si es una guía de cuestionamiento \\
se realiza de manera personal con cada estudiante y en & de lo aprendido, se revisa de manera oral con participación \\
otras asignaturas se realiza de la misma manera y en & abierta para que todos comenten los aciertos y errores al respec- \\
conjunto con el grupo." (Docente \#3- entrevistado) & to." (Docente \#1- entrevistado) \\
"Mediante tutorías en las asignaturas que finalizan con & "Si se aplicó una prueba y todos fallaron en el mismo ejercicio, \\
trabajos de curso." (Docente \#4- entrevistado) & realizamos un reforzamiento." (Docente \#2- entrevistado) \\
\hline
\end{tabular}

Ilustración 9: Fuente propia. Citas textuales de docentes 


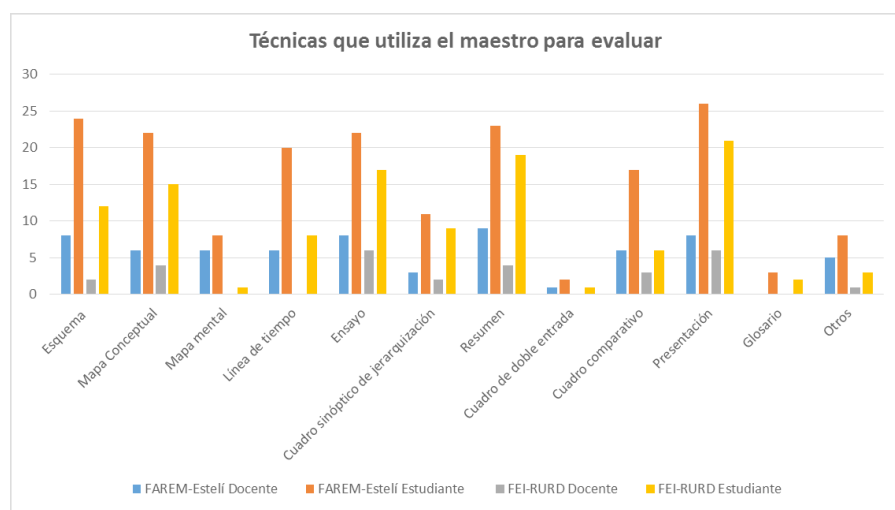

Ilustración 10: Fuente propia: Resultado de encuesta a docentes y estudiantes

En el proceso de aprendizaje, las técnicas de evaluación se utilizan con el propósito de desarrollar el pensamiento crítico, divergente y la metacognición; facilitando la gestión del conocimiento, mediante la investigación, la interpretación y la construcción de propuestas.

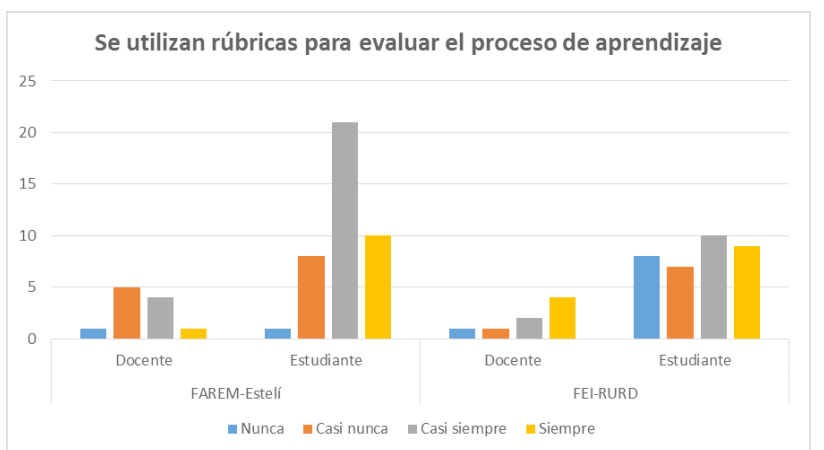

Ilustración 11: Fuente Propia: Resultado de encuesta de estudiantes y docentes.

Para la evaluación de aprendizajes, los implicados mencionan la utilización de rúbricas para la valoración de los resultados, sin embargo en la práctica la mayoría de los docentes no definen los instrumentos de evaluación que acompañen los procesos de aprendizaje, frente a los momentos de revisión (ensayo, línea del tiempo, presentaciones, resumen, entre otros); el estudiantado no tiene parámetros objetivos que les permita regular sus aprendizaje y por ende su rendimiento académico, sin embargo los criterios de evaluación de alguna manera son compartidos al momento de la presentación de los trabajos, como una práctica coyuntural. Con respecto a lo antes mencionado Cano (2012) plantea que las rúbricas, cuando son de conocimiento del estudiantado, contribuye a que perciban con mayor claridad los criterios por los que serán evaluados (P.71). 


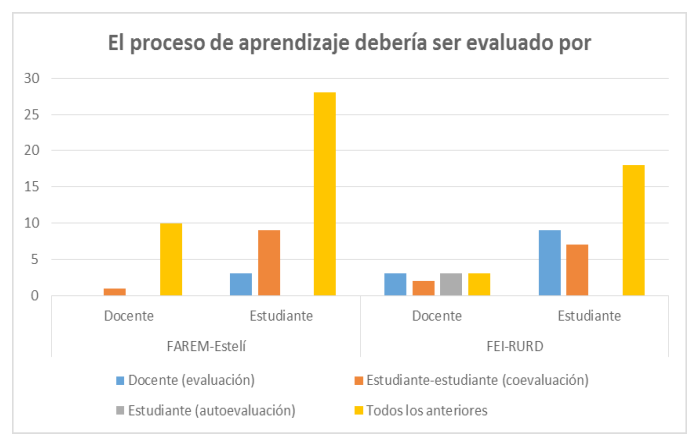

Ilustración 12: Fuente propia: Resultados de encuestas.

Docentes y estudiantes valoran que el proceso de aprendizaje debe ser evaluado tomando en cuenta los agentes involucrados; sin embargo, los docentes del RURD consideran que la evaluación es una responsabilidad exclusiva de ellos y raras veces involucra la autoevaluación y coevaluación, opiniones que contradice las características de la evaluación de ser un proceso democrático, compartido, negociado, desde la voz de los participantes como lo refiere López Mairena (2019 cita en Santos Guerra (1998), Barberá (1999) y Álvarez Méndez (1999), pp. 6-8).

Los docentes manifestaron, que en la práctica pedagógica la evaluación no parte de los intereses de los estudiantes, aunque se realizan acciones de información y comunicación donde se comparten generalidades de los procesos a desarrollar: contenidos, estrategias de aprendizajes y de evaluación.

"Siempre comparto con los grupos, pero no siempre les pido su opinión, depende, si el grupo en su mayoría es disciplinado lo hago en algunos casos. Pero, si el grupo es indisciplinado no lo hago ya que, debido a sus discrepancias, no se logra consenso entre ellos." (Docentes \#1- entrevistado)

"Desde el primer día, les propongo las estrategias de evaluación y consensuamos cuales de éstas se utilizarán. Por supuesto, algunas no son negociables como al menos una prueba escrita y el examen y/o trabajo de curso." (Docentes \#1- entrevistado)

La flexibilidad de la planificación didáctica depende de la práctica pedagógica y la visión de educación que asume cada docente en el proceso de aprendizaje; esto implica la desconstrucción de representaciones sociales que influyen en el rol docente, como es la misma concepción de evaluación, de estudiante, de aprendizaje, entre otros.

"Antes de entrar a los contenidos de la asignatura, si consulto con ellos y compartimos opiniones de cómo podemos realizar ese proceso de evaluación de manera que sea para mantener un buen ambiente." (Docentes \# 7- entrevistado) 


\section{Intervención educativa}

Se realizó en las modalidades presencial y virtual (grupo Facebook y Google Classroom), a través de encuentros de discusión, debate, reflexión y construcción colectiva de sensibilización teórica-práctica, oportunas para ser implementadas en los procesos de planificación didáctica.

Resultados de las actividades realizadas en los espacios virtuales

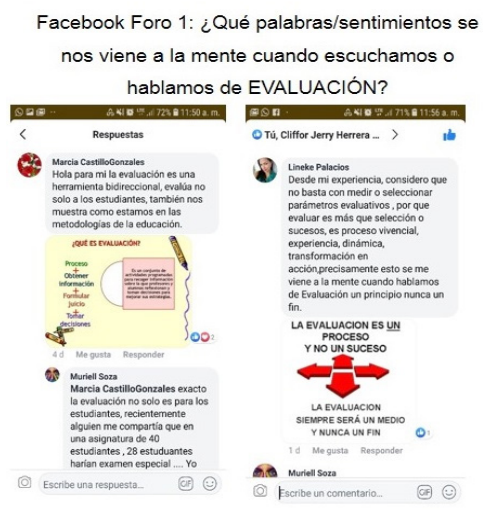

Facebook Foro 2: Cine Foro-Las patologías

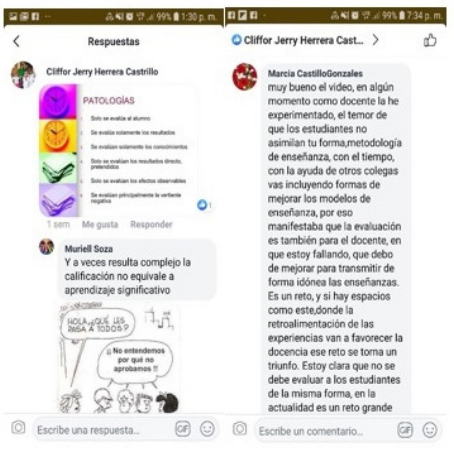

Ilustración 13: Fuente propia. Evidencia virtuales. Ilustración 14: Fuente propia. Evidencias virtuales.

Google Classroon: Taller de Docentes

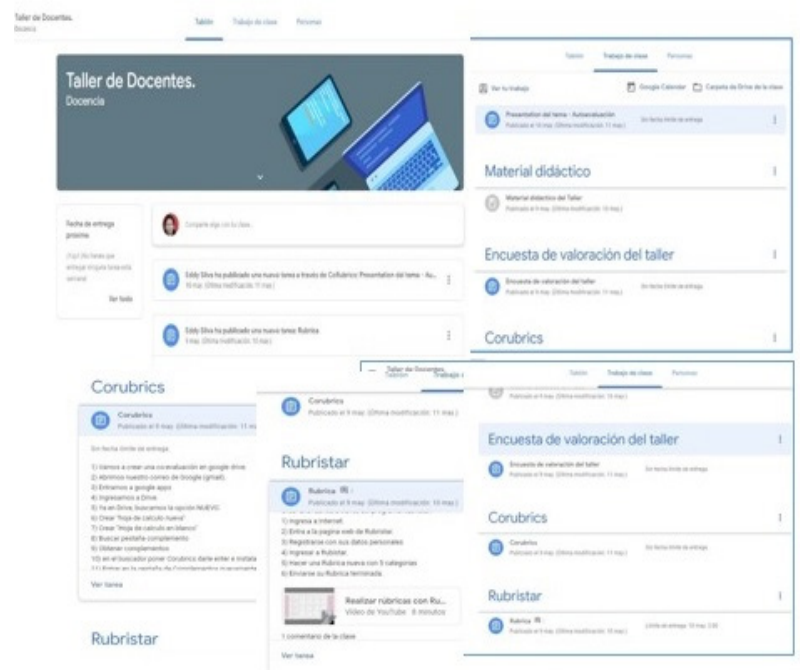

Ilustración 15: Fuente propia. Evidencia virtuales. 


\section{Discusión y Conclusiones}

En la práctica pedagógica, todavía predominan las concepciones tradicionales de las formas de evaluación, asumiendo la evaluación como un fin cuantificado, en lo general como una acción que mide y valora, descuidando la evaluación formativa y de proceso. En las aulas de clase el examen escrito es la estrategia de evaluación más utilizada.

Docentes y estudiantes, reconocen que los procesos de aprendizaje, requieren de estrategias de evaluación centradas en los estudiantes que potencien las relaciones humanas, horizontales e integrales, sin embargo en la práctica se priorizan las estrategias que no responden precisamente esos intereses.

Como UNAN- Managua, contamos con referentes institucionales y curriculares que orientan que la evaluación de los aprendizajes sea un proceso continuo, pertinente y contextualizado, donde los implicados son protagonistas de la construcción de aprendizajes para la vida; no obstante, la evaluación es realizada directamente por el docente.

Los docentes tienen dominio teórico de los diferentes tipos de evaluación (funcionalidad, temporalidad, según los agentes involucrados) sin embargo en su quehacer cotidiano no logran operativizar esos conocimientos en sus planeaciones didácticas, para desarrollar la gestión del conocimiento.

Los procesos de evaluación por su complejidad son asumidos como acciones estratégicas que solo responden a acciones sumativas, dejando de un lado el énfasis de la retroalimentación como una estrategia del desarrollo de aprendizajes.

Es importante para lograr la comprensión de la evaluación, que los docentes realicemos un proceso de transformación de los roles y la práctica, esto implica retomar a los estudiantes como el centro de la educación.

Las experiencias de investigación en el tema de evaluación deben de aportar al desarrollo de acciones innovadoras, creativas en donde docentes y estudiantes identifican las estrategias y formas de evaluación que permitan un aprendizaje significativo.

\section{Lista de referencias}

Amaranti Pesce, M. (2010). Evaluación de la educación. Concepciones y prácticas de retroalimentación de los profesores de lenguajes y comunicación de primer años educación media. Recuperado el 10 de mayo de 2019, de http://www.adeepra.org.ar/congresos/Congreso\%2oIBEROAMERICANO/EVALUACION/RLE2488_Amaranti.pdf 
Arias Lara, S., \& de Arias Peñaloza, M. (2011). valuar los aprendizajes: un enfoque innovador. Educere. Recuperado el 18 de abril de 2019, de http://www.redalyc. org/articulo.oa?id=35621559006

Cano, E. (2012). Aprobar o aprender. Estrategias de evaluación en la sociedad red. Barcelona: Colección Trasmedia XXI. Recuperado el 26 de abril de 2019, de http://www.lmi. ub.edu/transmedia21/pdf/4_AprobaroAprender.pdf

Fernández Marcha, A. (2009). Recuperado el 16 de mayo de 2019, de LA EVALUACIÓN DE LOS APRENDIZAJES EN LA UNIVERSIDAD LA EVALUACIÓN DE LOS APRENDIZAJES EN LA UNIVERSIDAD: NUEVOS ENFOQUES: https://web. ua.es/es/ice/documentos/recursos/materiales/ev-aprendizajes.pdf

Foronda Torrico, J., \& Foronda Zubieta, C. (2007). La evaluación en el proceso de aprendizaje. Perspectivas. Recuperado el 30 de abril de 2019, de http://www.redalyc. org/articulo.oa?id=35621559006

López Mairena, E., \& Trillo Alonso, J. (2013). Evaluación de las competencias en los estudiantes, URACCAN-Nueva Guinea. Ciencia E Interculturalidad, 12(1), 22-38. https://doi.org/https://doi.org/10.5377/rci.v12i1.1214

Najarro Arriola, A. (2009). Evaluación de los aprendizajes en la escuela primaria. una nueva visión. San Jose. Costa Rica: CECC/SICA. Recuperado el 10 de mayo de 2019, de http://ceccsica.info/sites/default/files/content/Volumen_21.pdf

Pimienta Prieto , J. (2008). Evaluación de los aprendizajes. Un enfoque basado en competencias. México: Pearson Educación. Recuperado el 23 de abril de 2019, de https://josedominguezblog.files.wordpress.com/2014/o8/evaluacic3b3n-delos-aprendizajes-enfoque-basado-en-competencias.pdf

Rodríguez Gómez , G., Ibarra Sáiz, M., \& García Jiménez, E. (2013). Autoevaluación, evaluación entre iguales y coevaluación: conceptualización y práctica en las universidades españolas. Revista de Investigación en Educación, 198-210. Recuperado el 26 de abril de 2019, de https://www.researchgate.net/publication/281677461_Autoevaluacion_evaluacion_entre_iguales_y_coevaluacion_conceptualizacion_y_practica_en_las_universidades_espanolas

Santos Guerra, M. (1999). 20 paradojas de la evaluación del alumnado en la Universidad española. Revista Electrónica Interuniversitaria de Formación de Profesorado. Recuperado el 2 de mayo de 2019, de https://www.aufop.com/aufop/uploaded_files/articulos/1224341617.pdf 
Tobón Tobón, S., Pimienta Prieto, J., \& García Fraile, J. (2010). Secuencias didácticas: aprendizaje y evaluación de competencias. México: PEARSON EDUCACIÓN. Recuperado el 13 de abril de 2019, de http://files.ctezona141.webnode.mx/2000000048edo38fca3/secuencias-didacticastobon-120521222400-phpappo2.pdf

UNAN-Managua. (2011). Modelo educativo, Normativa y Metodología para la Planificación Curricular. Managua. Recuperado el 20 de abril de 2019, de http://pagines.uab. cat/unan_uab_innovadocencia/sites/pagines.uab.cat.unan_uab_innovadocencia/files/Modelo_Educativo19_de_septiembre.pdf 